\title{
Existence of vertical spin stiffness in Landau-Lifshitz-Gilbert equation in ferromagnetic semiconductors
}

\author{
K. Shen,${ }^{1}$ G. Tatara, ${ }^{2}$ and M. W. Wu ${ }^{1, *}$ \\ ${ }^{1}$ Hefei National Laboratory for Physical Sciences at Microscale and Department of Physics, \\ University of Science and Technology of China, Hefei, Anhui, 230026, China \\ ${ }^{2}$ Department of Physics, Tokyo Metropolitan University, Hachioji, Tokyo 192-0397, Japan
}

(Dated: October 31, 2018)

\begin{abstract}
We calculate the magnetization torque due to the spin polarization of the itinerant electrons by deriving the kinetic spin Bloch equations based on the $s$ - $d$ model. We find that the first-order gradient of the magnetization inhomogeneity gives rise to the current-induced torques, which are consistent to the previous works. At the second-order gradient, we find an effective magnetic field perpendicular to the spin stiffness filed. This field is proportional to the nonadiabatic parameter $\beta$. We show that this vertical spin stiffness term can significantly modify the domain-wall structure in ferromagnetic semiconductors and hence should be included in the Landau-Lifshitz-Gilbert equation in studying the magnetization dynamics.
\end{abstract}

PACS numbers: 75.60.Ch, 72.25.Dc, 75.30.Gw, 75.50.Pp

\section{INTRODUCTION}

Ferromagnetic systems have attracted much interest for a long history because of the intriguing physics and applications $\stackrel{1,2}{=}$ As the development of information technology, the research on magnetization dynamics in micromagnets has become an active field $\underline{3}^{\underline{4}}$ Great efforts have been devoted to this field by aiming to manipulate magnetization more efficiently $\underline{\underline{\underline{5}} \underline{\underline{7}}}$ For theoretical simulation, the magnetization dynamics is usually described by the Landau-Lifshitz-Gilbert (LLG) equation,,$\frac{8,9}{9}$

$$
\dot{\mathbf{n}}=-\gamma \mathbf{n} \times \mathbf{H}_{\mathrm{eff}}+\alpha \mathbf{n} \times \dot{\mathbf{n}}-(1-\beta \mathbf{n} \times)\left(\mathbf{v}_{s} \cdot \nabla\right) \mathbf{n},
$$

where $\mathbf{n}$ represents the direction of the magnetization. $\mathbf{H}_{\text {eff }}$ in the first term on the right-hand side of Eq. (1) is the effective magnetic field which drives the magnetization procession and determines the domain structure in the equilibrium states. Up to date, different sources of effective magnetic field have been identified, e.g., the external magnetic field, the crystal anisotropy induced by the spin-orbit coupling, the exchange energy due to the spatial inhomogeneity, and also the demagnetization field from dipole-dipole interaction $\stackrel{10}{*}$ The second term, the Gilbert damping torque, describes the magnetization relaxation to the effective field axis on the time scale of $1 /\left(\alpha \gamma H_{\text {eff }}\right) \stackrel{8}{*}$ The last one with first-order gradient of the magnetization is the spin torque induced by the transport of the spin polarized itinerant electrons, where $\mathbf{v}_{s}$ is proportional to the spin current density $\underline{11}-17$ The $\beta$ term, $\stackrel{12,13}{1}$ first proposed by Zhang and $\mathrm{Li}, \underline{12}$ was demonstrated to be critically important to the current-driven domain wall motion, which overwhelms the threshold current due to pinning force and transverse anisotropy for domain wall motion $10,18,19$ and results in the steady domain wall velocity $\propto \beta / \alpha$ in the absence of the external magnetic field. Therefore, the determination of the effective magnetic field and the parameters in the LLG equation, such as $\alpha$ and $\beta$, is an important issue for magnetization dynamics study $\underline{12,14,15,20,21}$
Previously, we have derived the Gilbert damping $(\alpha)$ term based on the kinetic spin Bloch equation (KSBE) approach $^{22}$ in homogeneous ferromagnetic systems $\stackrel{21}{=}$ In the present work, we derive the whole LLG equation from the $s$ - $d$ model in inhomogeneous ferromagnetic systems based on the same approach. From the first-order gradient of the magnetization inhomogeneity, we obtain the current-induced torques which are consistent to the above LLG equation. Within the second-order gradient, we find that the LLG equation should be modified and written as

$$
\begin{aligned}
\dot{\mathbf{n}}= & -\gamma \mathbf{n} \times \mathbf{H}_{\mathrm{eff}}+\alpha \mathbf{n} \times \dot{\mathbf{n}}-(1-\beta \mathbf{n} \times)\left(\mathbf{v}_{s} \cdot \nabla\right) \mathbf{n} \\
& -\frac{\gamma}{M_{d}} A_{\mathrm{ss}} \mathbf{n} \times(1-\beta \mathbf{n} \times) \nabla^{2} \mathbf{n} .
\end{aligned}
$$

The second-order gradient introduces two contributions to the effective magnetic field. The one given by $A_{\mathrm{ss}} \nabla^{2} \mathbf{n}$ is identified as the spin stiffness field discussed in previous works $10,25,26$ The other term, in the form of $-\beta A_{\mathrm{ss}} \mathbf{n} \times \nabla^{2} \mathbf{n}$, has never be referred in the literature. In the present paper, we call it "vertical spin stiffness" in the sense of the fact that this new field is vertical to the plane defined by the magnetization and the normal spin stiffness $\nabla^{2} \mathbf{n}$. Interestingly, this vertical spin stiffness can not be written in terms of the free energy, and therefore, it can not be derived from the functional derivative of the free energy with respect to the local magnetization $\mathbf{H}_{\text {eff }}=-\delta F\left[\mathbf{M}_{d}\right] / \mathbf{M}_{d}$ previously ${ }^{9,10}$ We find that this vertical spin stiffness results in the tilt of the magnetization. The new term can significantly change the domain-wall structure in ferromagnetic semiconductors. Since the magnitude of this field is proportional to the factor of $\beta$, the proposed effect is expected to be important in ferromagnetic semiconductors where $\beta$ is large due to the strong spin-orbit interaction $\underline{\underline{20,23}}$

This paper is organized as follows: In Sec. II, we setup our model and derive the KSBEs for the itinerant electrons in the inhomogeneous ferromagnetic system. We calculate the spin torque by solving the KSBEs in Sec. III 
and discuss the results in Sec. IV. Finally, we briefly summarize in Sec. V.

\section{KSBES}

We use the exchange interaction Hamiltonian density $H_{\text {sd }}(\mathbf{r})=M \mathbf{n} \cdot \boldsymbol{\sigma}$ with $M$ denoting the coupling constant. Following Ref. 24, we here assume that the ferromagnetic interaction exists among $\mathbf{n}(\mathbf{r})$ and show later that this assumption is justified after integrating out the conduction electrons (giving rise to spin stiffness). The Pauli matrices $\boldsymbol{\sigma}$ are used to describe the itinerant electrons. In contrast to the previous work on the Gilbert damping, ${ }^{21}$ we introduce the inhomogeneity by considering the position dependence of the magnetization direction $\mathbf{n}(\mathbf{r})=\mathbf{M}_{d}(\mathbf{r}) / M_{d} . \quad M_{d}$ is the uniform saturate magnetization. For the strong exchange coupling in ferromagnetic system, the rotation framework ${ }^{4,15}$ is employed here. That is, the local spinor operators of the itinerant electrons are defined as $a(\mathbf{r})=\left(a_{\uparrow}(\mathbf{r}), a_{\downarrow}(\mathbf{r})\right)^{T}$, with $\uparrow(\downarrow)$ labeling the spin orientation parallel (antiparallel) to $\mathbf{n}(\mathbf{r})$. Therefore, one has $H_{\mathrm{sd}}=M a^{\dagger} \sigma_{z} a$. The spinor operators $a(\mathbf{r})$ are connected to the ones defined in the lattice coordinate system $c=\left(c_{\uparrow}, c_{\downarrow}\right)^{T}$ via the unitary transformation $a(\mathbf{r})=U(\mathbf{r}) c$. The transformation matrices is given by $U(\mathbf{r})=\mathbf{m}(\mathbf{r}) \cdot \boldsymbol{\sigma}$ with $\mathbf{m}=(\sin (\theta / 2) \cos \varphi, \sin (\theta / 2) \sin \varphi, \cos \theta)$ for $\mathbf{n}(\mathbf{r})=$ $(\sin \theta \cos \varphi, \sin \theta \sin \varphi, \cos \theta), \underline{4,15}$

In the rotation framework, the kinetic Hamiltonian should be expressed as $H_{\mathrm{k}}=|\nabla c|^{2} / 2 m=\mid(\nabla+$ $i \mathbf{A})\left.a\right|^{2} / 2 m=|\nabla a|^{2} / 2 m+H_{A}$, where the gauge field introduced by the coordinate transformation is given by $A_{i}=-i U^{\dagger} \partial_{i} U=\left(\mathbf{m} \times \partial_{i} \mathbf{m}\right)_{l} \sigma_{l}=A_{i}^{l} \sigma_{l}$. Then, one obtains the Hamiltonian density associated with the gauge field,,

$$
H_{A}=-\frac{i}{2 m} \sum_{i}\left[a^{\dagger} A_{i} \nabla_{i} a-\left(\nabla_{i} a\right)^{\dagger} A_{i} a\right]+a^{\dagger} \frac{A^{2}}{2 m} a
$$

To derive the KSBEs of the itinerant electrons, we follow the nonequilibrium Green function approach ${ }^{22,27,28}$ The Dyson equation of the contour-ordered Green function can be expressed as

$$
\begin{aligned}
G(1,2)= & G_{0}(1,2)+\int_{c} d 3 G_{0}(1,3) U_{e}(3) G(3,2) \\
& +\int_{c} d 3 \int_{c} d 4 G_{0}(1,3) \Sigma(3,4) G(4,2) \\
= & G_{0}(1,2)+\int_{c} d 3 G(1,3) U(3) G_{0}(3,2) \\
& +\int_{c} d 3 \int_{c} d 4 G(1,3) \Sigma(3,4) G_{0}(4,2),
\end{aligned}
$$

where the single particle contour-ordered Green function between two space-time points $(1)=\left(\mathbf{r}_{1}, t_{1}\right)$ and $(2)=\left(\mathbf{r}_{2}, t_{2}\right)$ on the contour $C$ is defined as $G(1,2)=$ $-i\left\langle T_{C}\left[\psi_{H}(1) \psi_{H}^{\dagger}(2)\right]\right\rangle \cdot{ }^{27} U_{e}$ describes the local electric potential energy, whereas $\Sigma$ represents the self-energy correction due to interactions, such as the electron-impurity, electron-phonon, and electron-electron interactions. $G_{0}$ stands for the free-particle Green function. The left- and right-inverses of $G_{0}(1,2)$ are given by

$$
\begin{aligned}
& \left(G_{0}\left(x_{1}, x_{2}\right)\right)_{x_{1}}^{-1}=i \partial_{t_{1}}-H_{0}\left(\mathbf{p}_{1}, \mathbf{r}_{1}\right) \\
& \left(\overleftarrow{G}_{0}\left(x_{1}, x_{2}\right)\right)_{x_{2}}^{-1}=-i \overleftarrow{\partial}_{t_{2}}-H_{0}\left(-\overleftarrow{\mathbf{p}}_{2}, \mathbf{r}_{2}\right)
\end{aligned}
$$

with $H_{0}=H_{\mathrm{k}}+H_{\mathrm{sd}}$. By multiplying them to Eq. (4), one obtains

$$
\begin{aligned}
& i\left(\partial_{t_{1}}+\partial_{t_{2}}\right) G(1,2)=\left[H_{0}\left(\mathbf{p}_{1}, \mathbf{r}_{1}\right)+U_{e}(1)\right] G(1,2) \\
& \quad-G(1,2)\left[H_{0}\left(-\overleftarrow{\mathbf{p}}_{2}, \mathbf{r}_{2}\right)+U_{e}(2)\right] \\
& \quad+\int_{c} d 3[\Sigma(1,3) G(3,2)-G(1,3) \Sigma(3,2)]
\end{aligned}
$$

with $\mathbf{p}_{i}$ corresponding to the momentum operators $-i \partial_{\mathbf{r}_{i}}$. To transform the above equation to the center-of-mass and relative variables

$$
\begin{aligned}
\mathbf{R} & =\left(\mathbf{r}_{1}+\mathbf{r}_{2}\right) / 2 ; \quad \mathbf{r}=\mathbf{r}_{1}-\mathbf{r}_{2} ; \\
T & =\left(t_{1}+t_{2}\right) / 2 ; \quad t=t_{1}-t_{2},
\end{aligned}
$$

one rewrites the following Green functions as

$$
\begin{aligned}
& G(1,2)=G\left(\mathbf{R}, \mathbf{r}, T+\frac{t}{2}, T-\frac{t}{2}\right), \\
& G(1,3)=e^{\frac{\mathbf{r}_{3}-\mathbf{r}_{2}}{2} \partial_{\mathbf{R}}} G\left(\mathbf{R}, \mathbf{r}_{1}-\mathbf{r}_{3}, T+\frac{t}{2}, t_{3}\right), \\
& G(3,2)=e^{\frac{\mathbf{r}_{3}-\mathbf{r}_{1}}{2} \partial_{\mathbf{R}}} G\left(\mathbf{R}, \mathbf{r}_{3}-\mathbf{r}_{2}, t_{3}, T-\frac{t}{2}\right) .
\end{aligned}
$$

The self-energy can be written in the same way. Similarly, one obtains

$$
\begin{aligned}
& U_{e}(1)=e^{\frac{\mathbf{r}}{2} \partial_{\mathbf{R}}} U_{e}\left(\mathbf{R}, T+\frac{t}{2}\right), \\
& U_{e}(2)=e^{-\frac{\mathbf{r}}{2} \partial_{\mathbf{R}}} U_{e}\left(\mathbf{R}, T-\frac{t}{2}\right) .
\end{aligned}
$$

The Hamiltonian can be written as

$$
\begin{aligned}
H_{0}\left(\mathbf{p}_{1}, \mathbf{r}_{1}\right) & =e^{\frac{\mathbf{r}}{2} \partial_{\mathbf{R}}^{H_{0}}} H_{0}\left(\frac{1}{2} \mathbf{P}_{\mathbf{R}}+\mathbf{p}, \mathbf{R}\right) \\
H_{0}\left(-\overleftarrow{\mathbf{p}}_{2}, \mathbf{r}_{2}\right) & =H_{0}\left(-\frac{1}{2} \overleftarrow{\mathbf{P}}_{\mathbf{R}}+\overleftarrow{\mathbf{p}}, \mathbf{R}\right) e^{-\frac{\mathbf{r}}{2}{\overleftarrow{\partial_{\mathbf{R}}}}^{H_{0}}}
\end{aligned}
$$

where $\mathbf{P}_{\mathbf{R}}$ and $\mathbf{p}$ represent the momentum operators respect to the center-of-mass and relative variables. According to Eq. (3), the left- and right-operators of the kinetic Hamiltonian should be different and can be written as

$$
\begin{aligned}
H_{\mathrm{k}}(\mathbf{p}, \mathbf{r}) & =\frac{1}{2 m}\left[\mathbf{p}^{2}-i(\nabla \cdot \mathbf{A})+2 \mathbf{A} \cdot \mathbf{p}+\mathbf{A}^{2}\right] \\
H_{\mathrm{k}}(-\overleftarrow{\mathbf{p}}, \mathbf{r}) & =\frac{1}{2 m}\left[\overleftarrow{\mathbf{p}}^{2}+i(\nabla \cdot \mathbf{A})-2 \overleftarrow{\mathbf{p}} \cdot \mathbf{A}+\mathbf{A}^{2}\right]
\end{aligned}
$$

separately. By assuming the magnetization varies smoothly respect to the spatial coordinates, we only keep the spatial gradient up to the second order. Since the gauge field is already the first-order gradient, both $\nabla \cdot \mathbf{A}$ and $\mathbf{A}^{2}$ are second-order gradient terms. We include both 
of them and neglect the higher-order ones. The gradient term of the gauge field can be written in the center-ofmass coordinate system as

$$
\begin{aligned}
\nabla_{\mathbf{r}_{1}} \cdot \mathbf{A}\left(\mathbf{r}_{1}\right) & =\frac{1}{2} \nabla_{\mathbf{R}} \cdot \mathbf{A}\left(\mathbf{R}+\frac{\mathbf{r}}{2}\right)+\nabla_{\mathbf{r}} \cdot \mathbf{A}\left(\mathbf{R}+\frac{\mathbf{r}}{2}\right) \\
& =\frac{1}{2} \nabla_{\mathbf{R}} \cdot \mathbf{A}(\mathbf{R})+\partial_{r_{i}} \frac{r_{j}}{2} \partial_{R_{j}} A^{i}(\mathbf{R}) \\
& =\nabla_{\mathbf{R}} \cdot \mathbf{A}(\mathbf{R}) .
\end{aligned}
$$

Similar calculation gives

$$
\nabla_{\mathbf{r}_{2}} \cdot \mathbf{A}\left(\mathbf{r}_{2}\right)=\nabla_{\mathbf{R}} \cdot \mathbf{A}(\mathbf{R}) .
$$

Moreover, one can easily show that $\mathbf{A}^{2}\left(\mathbf{r}_{i}\right) \approx \mathbf{A}^{2}(\mathbf{R})=$ $\mathbf{I} \sum_{i, l}\left(A_{i}^{l}\right)^{2}$ with $\mathbf{I}$ representing the unit matrix.

By substituting all these equations into Eq. (7) and doing Fourier transformation respect to the relative coordinate $\mathbf{r}$, one obtains

$$
\begin{aligned}
& i \partial_{T} G\left(\mathbf{R}, \mathbf{k}, t_{1}, t_{2}\right) \\
&=e^{i \frac{1}{2}\left(\partial_{\mathbf{k}} \partial_{\mathbf{R}}^{H_{0}}-\partial_{\mathbf{R}}^{G} \partial_{\mathbf{k}}^{H_{0}}\right)} H_{0}(\mathbf{k}, \mathbf{R}) G\left(\mathbf{R}, \mathbf{k}, t_{1}, t_{2}\right) \\
& \quad-e^{-i \frac{1}{2}\left(\partial_{\mathbf{k}} \partial_{\mathbf{R}}^{H_{0}}-\partial_{\mathbf{R}}^{G} \partial_{\mathbf{k}}^{H_{0}}\right)} G\left(\mathbf{R}, \mathbf{k}, t_{1}, t_{2}\right) H_{0}(\mathbf{k}, \mathbf{R}) \\
& \quad+e^{i \frac{1}{2} \partial_{\mathbf{k}}^{G} \partial_{\mathbf{R}}^{U_{e}}} U_{e}\left(\mathbf{R}, t_{1}\right) G\left(\mathbf{R}, \mathbf{k}, t_{1}, t_{2}\right) \\
& \quad-e^{-i \frac{1}{2} \partial_{\mathbf{k}}^{G} \partial_{\mathbf{R}}^{U_{e}} G\left(\mathbf{R}, \mathbf{k}, t_{1}, t_{2}\right) U_{e}\left(\mathbf{R}, t_{2}\right)} \\
&+\int_{c} d t_{3}\left[e^{\frac{i}{2}\left(\partial_{\mathbf{k}}^{G} \partial_{\mathbf{R}}^{\Sigma}-\partial_{\mathbf{k}}^{\Sigma} \partial_{\mathbf{R}}^{G}\right)} \Sigma\left(\mathbf{R}, \mathbf{k}, t_{1}, t_{3}\right) G\left(\mathbf{R}, \mathbf{k}, t_{3}, t_{2}\right)\right. \\
&-\left.e^{\frac{i}{2}\left(\partial_{\mathbf{k}}^{\Sigma} \partial_{\mathbf{R}}^{G}-\partial_{\mathbf{k}}^{G} \partial_{\mathbf{R}}^{\Sigma}\right)} G\left(\mathbf{R}, \mathbf{k}, t_{1}, t_{3}\right) \Sigma\left(\mathbf{R}, \mathbf{k}, t_{3}, t_{2}\right)\right] .
\end{aligned}
$$

The details can be found in Appendix A. We then perform the gradient expansion up to the first order and obtain

$$
\begin{aligned}
i \partial_{T} G= & {\left[H_{0}+U_{e}, G\right]-\frac{i}{2}\left\{\partial_{\mathbf{k}} H_{0}, \partial_{\mathbf{R}} G\right\}+\frac{i}{2}\left\{\partial_{\mathbf{R}} \partial_{\mathbf{k}} H_{0}, G\right\} } \\
& +\frac{i}{2}\left\{\partial_{\mathbf{R}}\left(H_{0}+U_{e}\right), \partial_{\mathbf{k}} G\right\}+\int_{c} d t_{3}(\Sigma G-G \Sigma),(21)
\end{aligned}
$$

where all the quantities are defined at $\mathbf{R}$ and $\mathbf{k}$. We should point out that the commutator notation $\left[H_{0}+\right.$ $\left.U_{e}, G\right]$ is still used although the left- and right-operators of $H_{0}$ are in different expressions [see Eqs. (16) and (17)]. By taking the isochronous condition, i.e., $t \rightarrow 0$, one has 27

$$
\begin{aligned}
& \int_{c} d t_{3}[\Sigma(T, \tau) G(\tau, T)-G(T, \tau) \Sigma(\tau, T)]^{<} \\
& =\int_{-\infty}^{T} d \tau\left[\Sigma^{>}(T, \tau) G^{<}(\tau, T)-\Sigma^{<}(T, \tau) G^{>}(\tau, T)\right. \\
& \left.-G^{>}(T, \tau) \Sigma^{<}(\tau, T)+G^{<}(T, \tau) \Sigma^{>}(\tau, T)\right],
\end{aligned}
$$

where the lesser and greater Green functions are defined by $G^{<}\left(\tau, \tau^{\prime}\right)=i\left\langle\psi_{H}^{\dagger}\left(\tau^{\prime}\right) \psi_{H}(\tau)\right\rangle$ and $G^{>}\left(\tau, \tau^{\prime}\right)=$ $-i\left\langle\psi_{H}(\tau) \psi_{H}^{\dagger}\left(\tau^{\prime}\right)\right\rangle$. Therefore, the correlation function $G^{<}(T, T)$ can be written as

$$
\begin{aligned}
i \partial_{T} G^{<}= & \int_{-\infty}^{T} d \tau\left(\Sigma^{>} G^{<}-\Sigma^{<} G^{>}-G^{>} \Sigma^{<}+G^{<} \Sigma^{>}\right) \\
& +\frac{i}{2}\left\{\partial_{\mathbf{R}} \partial_{\mathbf{k}} H_{0}, G^{<}\right\}+\frac{i}{2}\left\{\partial_{\mathbf{R}}\left(H_{0}+U_{e}\right), \partial_{\mathbf{k}} G^{<}\right\} \\
& +\left[H_{0}+U_{e}, G^{<}\right]-\frac{i}{2}\left\{\partial_{\mathbf{k}} H_{0}, \partial_{\mathbf{R}} G^{<}\right\} .
\end{aligned}
$$

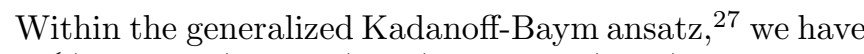
$G^{<}(\mathbf{R}, \mathbf{k}, T, T)=i \rho_{\mathbf{k}}(\mathbf{R}, T)$ where $\rho_{\mathbf{k}}(\mathbf{R}, T)$ is the local density matrix of the itinerant electrons with momentum $\mathbf{k}$ located at $\mathbf{R}$. Therefore, we write the general form of the KSBEs of the itinerant electrons as

$$
\begin{aligned}
& \partial_{t} \rho_{\mathbf{k}}+i\left[H_{0}, \rho_{\mathbf{k}}\right]+\frac{1}{2}\left\{\nabla_{\mathbf{k}} H_{0}, \nabla_{\mathbf{R}} \rho_{\mathbf{k}}\right\}-\nabla_{\mathbf{R}} U_{e} \cdot \nabla_{\mathbf{k}} \rho_{\mathbf{k}} \\
& -\frac{1}{2}\left\{\nabla_{\mathbf{R}} H_{0}, \nabla_{\mathbf{k}} \rho_{\mathbf{k}}\right\}-\frac{1}{2}\left\{\nabla_{\mathbf{R}} \cdot \nabla_{\mathbf{k}} H_{0}, \rho_{\mathbf{k}}\right\} \\
& =\left.\partial_{t} \rho_{\mathbf{k}}\right|_{\text {scat }} ^{c}+\left.\partial_{t} \rho_{\mathbf{k}}\right|_{\text {scat }} ^{f} .
\end{aligned}
$$

On the right-hand side of above equations, $\left.\partial_{t} \rho_{\mathbf{k}}\right|_{\text {scat }} ^{c}$ and $\left.\partial_{t} \rho_{\mathbf{k}}\right|_{\text {scat }} ^{f}$ from the integral term in Eq. (23) represent the spin-conserving and spin-flip scatterings. The details of these terms can be found in Ref.22.

We specify the Hamiltonian $H_{0}=M \sigma_{z}+\left[\left(k^{2}+2 A_{i} k_{i}+\right.\right.$ $\left.\left.A_{i}^{l} A_{i}^{l}\right) \mathbf{I} \mp i(\nabla \cdot \mathbf{A})\right] /(2 m)$ with the upper (lower) sign representing the left (right) operator case. The electric potential energy is given by $U_{e}=e \mathbf{E} \cdot \mathbf{R}(e>0)$. The final form of the KSBEs is given by

$$
\begin{aligned}
& \partial_{t} \rho_{\mathbf{k}}+i\left[M \sigma_{z}, \rho_{\mathbf{k}}\right]+i \frac{k_{i}}{m}\left[A_{i}, \rho_{\mathbf{k}}\right]+\frac{1}{2 m}\left\{\mathbf{A}, \nabla_{\mathbf{R}} \rho_{\mathbf{k}}\right\} \\
& +\frac{1}{m} \mathbf{k} \cdot \nabla_{\mathbf{R}} \rho_{\mathbf{k}}-e \mathbf{E} \cdot \nabla_{\mathbf{k}} \rho_{\mathbf{k}}-\frac{k_{i}}{2 m}\left\{\nabla_{\mathbf{k}} \rho_{\mathbf{k}}, \nabla_{\mathbf{R}} A_{i}\right\} \\
& =\left.\partial_{t} \rho_{\mathbf{k}}\right|_{\text {scat }} ^{c}+\left.\partial_{t} \rho_{\mathbf{k}}\right|_{\text {scat }} ^{f} .
\end{aligned}
$$

Interestingly, we find that the contribution from $-\frac{1}{2}\left\{\nabla_{\mathbf{r}}\right.$. $\left.\nabla_{\mathbf{k}} H_{0}, \rho_{\mathbf{k}}\right\}$ is completely canceled by the gauge field gradient term from $i\left[H_{0}, \rho_{\mathbf{k}}\right]$ and the $\mathbf{A}^{2}$-term is irrelevant.

\section{SOLUTION OF KSBES}

In general cases, the KSBEs are too complicated to solve analytically and the numerical scheme should be employed. However, the analytical solution can still be expected within some simplification of the KSBEs. In the following, we assume: $(i)$ the spatial dependence is weak in the rotation coordinate systems, hence $\nabla_{\mathbf{R}} \rho_{\mathbf{k}} \approx 0$; (ii) the scattering is strong enough to set up the steadystate condition $\partial_{t} \rho_{\mathbf{k}} \approx 0$. Without loss of generality, we take the magnetization gradient along arbitrary direction. The external electric field is applied on the purpose of producing current-induced magnetization dynamics. Therefore, the KSBEs become

$$
\begin{aligned}
& -e \mathbf{E} \cdot \nabla_{\mathbf{k}} \rho_{\mathbf{k}}+i\left[\frac{k_{i}}{m} A_{i}+M \sigma_{z}, \rho_{\mathbf{k}}\right]-\frac{k_{i}}{2 m}\left\{\nabla_{\mathbf{k}} \rho_{\mathbf{k}}, \nabla_{\mathbf{R}} A_{i}\right\} \\
& =\left.\partial_{t} \rho_{\mathbf{k}}\right|_{\text {scat }} ^{c}+\left.\partial_{t} \rho_{\mathbf{k}}\right|_{\text {scat }} ^{f} .
\end{aligned}
$$

For the steady-state situation with a small current due to a static electric field, we assume that the distribution of the itinerant electrons is not far away from the Fermi distribution. The scattering effect of the spin-conserving process is introduced by the relaxation time approximation with the average momentum relaxation time $\tau$. Therefore, one can linearly expand the density matrices by considering the drift effect, $\rho_{\mathbf{k}}=\rho_{\mathbf{k}}^{0} I+\mathbf{S}_{\mathbf{k}} \cdot \boldsymbol{\sigma}=$ $\rho_{k}^{i}+e \tau \mathbf{E} \cdot \nabla_{\mathbf{k}} \rho_{\mathbf{k}}^{i}$, where the isotropic density matrices $\rho_{k}^{i}=\rho_{k}^{i, 0} \mathbf{I}+\mathbf{S}_{k}^{i} \cdot \boldsymbol{\sigma}$ representing the spin polarized Fermi 
distribution in the absence of the electric field. By substituting these density matrices into Eq. (26), the driving term and the spin-conserving scattering term cancel out. Further, one introduces the average spin relaxation time $\tau_{s}$ and rewrite the spin-flip scattering as $-\frac{\left(\mathbf{S}_{\mathbf{k}}-\mathbf{S}_{\mathbf{k}}^{e}\right) \cdot \boldsymbol{\sigma}}{\tau_{s}}$. Here, $\mathbf{S}_{\mathbf{k}}^{e}$ describes the equilibrium spin polarization due to the spin-splitted band structure in the ferromagnetic system.

Under the above procedures, one finally obtains the equations of the steady-state spin polarization

$$
-\left(2 \mathbf{M}+\frac{2 k_{i}}{m} \mathbf{A}_{i}\right) \times \mathbf{S}_{\mathbf{k}}-\frac{k_{i}}{m} \nabla_{\mathbf{k}} \rho_{\mathbf{k}}^{0} \cdot \nabla_{\mathbf{R}} \mathbf{A}_{i}=-\frac{\mathbf{S}_{\mathbf{k}}-\mathbf{S}_{\mathbf{k}}^{e}}{\tau_{s}},
$$

by using the relation $\left\{\nabla_{\mathbf{k}} \rho_{\mathbf{k}}, \nabla_{\mathbf{R}} A_{i}\right\}=2 \nabla_{\mathbf{k}} \rho_{\mathbf{k}}^{0}$. $\nabla_{\mathbf{R}} A_{i}^{\beta} \sigma_{\beta}+2 \nabla_{\mathbf{k}} S_{\mathbf{k}}^{\alpha} \cdot \nabla_{\mathbf{R}} A_{i}^{\alpha}$. Here, we denotes $\mathbf{A}_{i}=$ $\left(A_{i}^{x}, A_{i}^{y}, A_{i}^{z}\right)$.

Then, the equation of the total spin polarization $\mathbf{S}$ can be obtained by summing Eq. (27) over the k-space

$$
\begin{aligned}
-2 \mathbf{M} & \times \mathbf{S}-\frac{2}{m} \mathbf{A}_{i} \times\left(\sum_{\mathbf{k}} k_{i} \mathbf{S}_{\mathbf{k}}\right) \\
& =-\frac{\mathbf{S}-\mathbf{S}^{e}}{\tau_{s}}+\frac{1}{m}\left(\sum_{\mathbf{k}} k_{i} \nabla_{\mathbf{k}} \rho_{\mathbf{k}}^{0}\right) \cdot \nabla_{\mathbf{R}} \mathbf{A}_{i} .
\end{aligned}
$$

For the lowest order approximation, one substitutes $\mathbf{S}_{\mathbf{k}}=$ $\mathbf{S}_{k}^{i}+e \tau \mathbf{E} \cdot \nabla_{\mathbf{k}} \mathbf{S}_{\mathbf{k}}^{i}$ into Eq. (28). By considering $\sum_{\mathbf{k}} k_{i} \mathbf{S}_{\mathbf{k}}^{i}=$ 0 , one obtains

$$
\mathbf{S}-2 \tau_{s} \mathbf{M} \times \mathbf{S}+2 \tau_{s} \mathbf{A}_{i} \times\left(\frac{1}{m} e \tau E_{i} \mathbf{S}\right)=\mathbf{S}^{e}-\tau_{s} \frac{n}{2 m} \partial_{i} \mathbf{A}_{i},
$$

in which the relation $\sum_{\mathbf{k}} k_{i} \partial_{k_{j}} g(\mathbf{k})=-\delta_{i j} \sum_{\mathbf{k}} g(\mathbf{k})$ is used. The quantity $n=2 \sum_{\mathbf{k}} \rho_{\mathbf{k}}^{0}$ stands for the density of the itinerant electrons. The spin polarization can then be found

$$
\mathbf{S}=\frac{\mathbf{y}+\mathcal{A} \times \mathbf{y}+(\mathcal{A} \cdot \mathbf{y}) \mathcal{A}}{1+|\mathcal{A}|^{2}}
$$

where $\mathcal{A}=2 \tau_{s} \mathbf{M}-2 \tau_{s} \tau \frac{e}{m} E_{i} \mathbf{A}_{i}$ and $\mathbf{y}=\mathbf{S}^{e}-\tau_{s} \frac{n}{2 m} \partial_{i} \mathbf{A}_{i}$. The equilibrium spin polarization $\mathbf{S}^{e}=\frac{1}{2} P n \hat{\mathbf{z}}$ with the value of the spin polarizability along the magnetization direction $P$ is negative, because $\mathbf{S}^{e}$ is anti-parallel to $\mathbf{M}_{d}$ and $\mathbf{M}$. With the current defined as $\mathbf{j}=e^{2} n \mathbf{E} / m$, the transverse spin polarization is given by

$$
\begin{aligned}
\mathbf{S}^{\perp} \approx & \left(1+4 \tau_{s}^{2} M^{2}\right)^{-1}\left(-\frac{n \tau_{s}}{2 m} \partial_{i} \mathbf{A}_{i}^{\perp}-\frac{M n \tau_{s}^{2}}{m} \hat{\mathbf{z}} \times \partial_{i} \mathbf{A}_{i}^{\perp}\right. \\
& \left.+\frac{\tau_{s} P}{e} j_{i} \hat{\mathbf{z}} \times \mathbf{A}_{i}^{\perp}-\frac{2 M \tau_{s}^{2} P}{e} j_{i} \mathbf{A}_{i}^{\perp}\right)
\end{aligned}
$$

Here, we have neglected the gauge field in the denominator. We should point out that the subscript $i$ refers to the lattice coordinate axis. In contrast, the gauge field is defined in the rotation frame, hence one needs to transform the relevant terms back to the lattice coordinate system. The rotation transformations are given by $R \mathbf{A}_{\mu}^{\perp}=-\frac{1}{2} \mathbf{n} \times \partial_{\mu} \mathbf{n}$ and $R\left(\hat{\mathbf{z}} \times \mathbf{A}_{\mu}^{\perp}\right)=\frac{1}{2} \partial_{\mu} \mathbf{n} .{ }^{15}$ One then obtains the transverse spin polarization in the lattice coordinate

$$
\begin{aligned}
\mathbf{S}_{l}^{\perp} \approx & \left(1+4 \tau_{s}^{2} M^{2}\right)^{-1}\left[\frac{n \tau_{s}}{4 m} \mathbf{n} \times \nabla^{2} \mathbf{n}-\frac{M n \tau_{s}^{2}}{2 m} \nabla^{2} \mathbf{n}\right. \\
& \left.+\frac{\tau_{s} P}{2 e}(\mathbf{j} \cdot \nabla) \mathbf{n}+\frac{M \tau_{s}^{2} P}{e} \mathbf{n} \times(\mathbf{j} \cdot \nabla) \mathbf{n}\right]
\end{aligned}
$$

Since the $s$ - $d$ exchange interaction can be equivalently written as $H_{s d}=\mathbf{M} \cdot\langle\boldsymbol{\sigma}\rangle=\frac{2 M}{M_{d}} \mathbf{M}_{d} \cdot \mathbf{S}$, the spin procession field induced by the $s$ - $d$ exchange interaction $-2 M \mathbf{S}_{l} / M_{d}$ is

$$
\begin{aligned}
\mathbf{H}^{\perp}= & \frac{1}{M_{d}}\left(1+\beta^{2}\right)^{-1}\left[\frac{n}{4 m}(1-\beta \mathbf{n} \times) \nabla^{2} \mathbf{n}\right. \\
& \left.-\frac{P}{2 e}(\beta+\mathbf{n} \times)(\mathbf{j} \cdot \nabla) \mathbf{n}\right],
\end{aligned}
$$

with $\beta=1 /\left(2 M \tau_{s}\right)$.

We finally obtain the LLG equation with Gilbert damping torque as Eq. (2), where $\mathbf{v}_{s}=\mathbf{j} P /\left[2 e S_{d}\left(1+\beta^{2}\right)\right]$ and

$$
A_{\mathrm{ss}}=n /\left[4 m\left(1+\beta^{2}\right)\right] \text {. }
$$

Here, the effective magnetic field $\mathbf{H}_{\text {eff }}$ includes the isotropic and demagnetization sources ${ }^{26}$ as well as the external magnetic field.

\section{DISCUSSION}

We discuss our results based on Eq. (2). One notices that the third term on the right-hand side of the equation is the current-induced torque obtained in the previous works. $12,14,15$ The fourth term is independent of the current but associated to the second-order gradient of the magnetization. This term contains two contributions. The one in the form $\frac{1}{M_{d}} A_{\mathrm{ss}} \nabla^{2} \mathbf{n}$ is identified as the effective spin stiffness. $^{25}$ In the limit $\beta \ll 1$ or $M \tau_{s} \gg 1$, from Eq. (34) one has $A_{\mathrm{ss}}=n /(4 m)$, which is consistent with the previous result ${ }^{25}$ However, $A_{\mathrm{ss}}$ should be modified for finite $\beta$. This stiffness is widely used in the study on the domain wall ${ }^{10,12}$ and demonstrated to be critical to determine the width of the domain wall.$^{29}$ One finds that the spin stiffness increases with increasing the exchange coupling strength $M$, which agrees with the previous computation. ${ }^{26}$ By taking $n \sim 10^{20} \mathrm{~cm}^{-3}$, $\beta \sim 1$ (Ref. 20 ), and $m=0.5 m_{e}$ with $m_{e}$ representing the free electron mass, one estimates the spin stiffness $A_{\mathrm{ss}} \sim 1 \mathrm{pJ} / \mathrm{m}$ in GaMnAs${ }^{26}$ However, the other effective field in the form $-\frac{\beta}{M_{d}} \mathbf{n} \times \nabla^{2} \mathbf{n}$ has not been studied yet. It is obvious that this torque prevents the magnetization from varying in a plane and induces transverse component instead. One notices that this term has no contribution to the free energy, because it is always perpendicular to the magnetization. Therefore, it can not be derived from the variation of the free energy with respect to the magnetization, which can explain the reason for missing this term in previous works. In the following, we focus on the steady domain-wall solution of the LLG equation in the absence of the current to illustrate the effect of this new effective magnetic field due to the vertical spin stiffness.

In the ferromagnetic thin film or nanowire structures, one takes the total effective magnetic field, $\mathbf{H}_{\text {eff }}^{\text {tot }}=$ $K n_{x} \hat{\mathbf{x}}-K_{\perp} n_{z} \hat{\mathbf{z}}+A_{\text {eff }} \nabla^{2} \mathbf{n}-\beta^{\prime} A_{\text {eff }} \mathbf{n} \times \nabla^{2} \mathbf{n}, 10$ with $K$ and $K_{\perp}$ representing the anisotropy constant and 
demagnetization field, respectively. Here, we have added stiffness constant $A_{\mathrm{ss}}^{0}$ arising from the nonitinerant-electron origin (such as dipole-dipole interaction between the localized $d$ electrons) to describe general systems, $A_{\mathrm{eff}}=\left(A_{\mathrm{ss}}+A_{\mathrm{ss}}^{0}\right) / M_{d}$ and $\beta^{\prime}=$ $\beta A_{\mathrm{ss}} /\left(A_{\mathrm{eff}} M_{d}\right)$. The magnetization direction is given by $\mathbf{n}=(\cos \theta, \sin \theta \cos \varphi, \sin \theta \sin \varphi)$. Obviously, the ground state is the homogeneous configuration with the magnetization pointing to the easy axis, i.e., the $\pm \hat{\mathbf{x}}$-axis. However, the inhomogeneous configurations can also stably exist, for example the domain wall structure. To discuss the formation of the inhomogeneous magnetization structure, we first write the equation of motion for $\theta$ and $\varphi$ in the absence of the current,

$$
\begin{aligned}
\dot{\theta}+\alpha \sin \theta \dot{\varphi}= & -\gamma K_{\perp} \sin \theta \sin \varphi \cos \varphi+\frac{\gamma A_{\text {eff }}}{\sin \theta} \partial_{x}\left(\sin ^{2} \theta \partial_{x} \varphi\right) \\
& -\gamma \beta^{\prime} A_{\text {eff }}\left[\partial_{x}^{2} \theta-\sin \theta \cos \theta\left(\partial_{x} \varphi\right)^{2}\right], \\
-\alpha \dot{\theta}+\sin \theta \dot{\varphi}= & \gamma K_{\perp} \sin \theta \cos \theta \sin ^{2} \varphi+\gamma K \sin \theta \cos \theta \\
& -\gamma A_{\text {eff }}\left[\partial_{x}^{2} \theta-\sin \theta \cos \theta\left(\partial_{x} \varphi\right)^{2}\right] \\
& -\frac{\gamma \beta^{\prime} A_{\text {eff }}}{\sin \theta} \partial_{x}\left(\sin ^{2} \theta \partial_{x} \varphi\right) .
\end{aligned}
$$

For the steady state, one obtains

$$
\begin{aligned}
\partial_{x}^{2} \theta= & {\left[\frac{K}{A_{\text {eff }}} \sin \theta \cos \theta+\frac{K_{\perp}}{A_{\text {eff }}} \sin \theta \sin \varphi\left(\cos \theta \sin \varphi-\beta^{\prime} \cos \varphi\right)\right.} \\
& \left.+\left(1+{\beta^{\prime}}^{2}\right) \sin \theta \cos \theta\left(\partial_{x} \varphi\right)^{2}\right] /\left(1+{\beta^{\prime}}^{2}\right) \\
\partial_{x}^{2} \varphi= & {\left[\frac{\beta^{\prime} K}{A_{\text {eff }}} \cos \theta+\frac{K \perp}{A_{\text {eff }}} \sin \varphi\left(\beta^{\prime} \cos \theta \sin \varphi+\cos \varphi\right)\right.} \\
& \left.-\left(1+{\beta^{\prime}}^{2}\right) 2 \cot \theta \partial_{x} \theta \partial_{x} \varphi\right] /\left(1+{\beta^{\prime}}^{2}\right) .
\end{aligned}
$$

At $\beta^{\prime}=0$, one obtains a single wall solution in the $x-y$ plane (located at $\left.x=x_{0}\right), \varphi=n \pi, \ln \left(\tan \frac{\theta}{2}\right)=\frac{x-x_{0}}{W}$ with $W=\sqrt{A_{\text {eff }} / K}$.

When $\beta^{\prime} \neq 0$, the magnetization can not vary in a fixed plane since $\theta$ and $\varphi$ are coupled. Unfortunately, Eqs. (36) and (37) cannot be solved analytically in general. However, in the absence of the demagnetization field $\left(K_{\perp}=0\right)$, there is a solution where the gradient of $\varphi$ is a constant, i.e., $\partial_{x} \varphi=\lambda$. In this case, the equations can be written as

$$
\begin{aligned}
\partial_{x}^{2} \theta & =\frac{1}{\left(1+\beta^{\prime 2}\right)}\left[\frac{K}{A_{\text {eff }}} \sin \theta \cos \theta+\lambda^{2}\left(1+{\beta^{\prime}}^{2}\right) \sin \theta \cos \theta\right](39) \\
0 & =\frac{1}{\left(1+\beta^{\prime 2}\right)}\left[\frac{\beta^{\prime} K}{A_{\text {eff }}} \cos \theta-\lambda\left(1+{\beta^{\prime}}^{2}\right) 2 \cot \theta \partial_{x} \theta\right] .
\end{aligned}
$$

Obviously, both equations give the solution in the same form

$$
\ln \left(\tan \frac{\theta}{2}\right)=\frac{x-x_{0}}{W_{h}},
$$

which is just the domain wall solution with the corresponding width $W_{h}^{a}=\sqrt{\left(1+{\beta^{\prime}}^{2}\right) /\left[\frac{K}{A_{\text {eff }}}+\left(1+{\beta^{\prime}}^{2}\right) \lambda^{2}\right]}$ and $W_{h}^{b}=2|\lambda| A_{\text {eff }}\left(1+{\beta^{\prime}}^{2}\right) /\left(\beta^{\prime} K\right)$, respectively. The self-consistent condition $W_{h}^{a}=W_{h}^{b}$ determines the value of $\lambda$ as

$$
\lambda= \pm \sqrt{\frac{K}{A_{\text {eff }}}}\left[\frac{\sqrt{1+\beta^{\prime 2}}-1}{2\left(1+\beta^{\prime 2}\right)}\right]^{\frac{1}{2}} .
$$
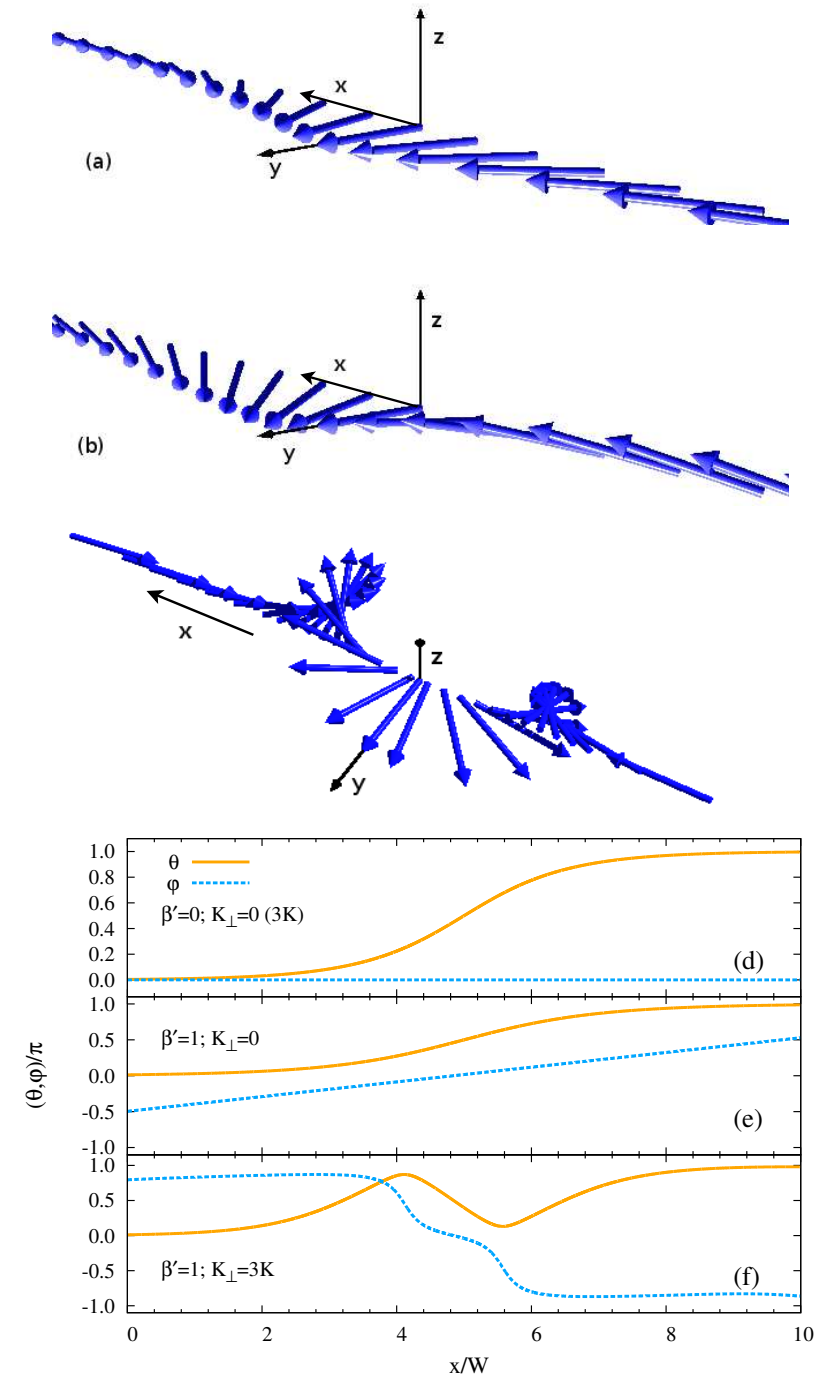

FIG. 1: (Color Online) The variation of the direction of magnetization along the $x$-axis is plotted as: (a) $\beta^{\prime}=0$, $K_{\perp}=0($ or $3 K)$; (b) $\beta^{\prime}=1, K_{\perp}=0$; and (c) $\beta^{\prime}=1$ and $K_{\perp}=3 K$. By denoting the magnetization direction $\mathbf{n}=(\cos \theta, \sin \theta \cos \varphi, \sin \theta \sin \varphi)$, the position dependences of $\theta$ and $\varphi$ are given in (d)-(f). $W=\sqrt{A_{\text {eff }} / K}$.

The domain wall thickness is enhanced by $\beta^{\prime}$ as

$$
W_{h}=\sqrt{\frac{A_{\text {eff }}}{K} \frac{2\left(1+{\beta^{\prime}}^{2}\right)}{1+\sqrt{1+\beta^{\prime 2}}}} .
$$

This solution indicates that the magnetization rotates along the easy axis inside the domain wall. One can calculate the change of $\varphi$ across the domain wall to obtain $\Delta \varphi=\lambda W_{h}=\left(\sqrt{1+{\beta^{\prime}}^{2}}-1\right) / \beta^{\prime}$. When $\beta^{\prime} \ll 1$, one finds that $\Delta \varphi=\beta^{\prime} / 2 \ll 1$, which indicates that $\varphi$ can be approximated as a constant within a domain wall as shown in Fig.1(a), where the magetization always lies in the $x-y$ plane [see also Fig.11(d)]. The solution, therefore, returns to the Néel wall case $\frac{12}{2}$ In contrast, when 
$\beta^{\prime} \gtrsim 1, \varphi$ oscillates within a wall and the wall structure becomes more like a one-dimensional vortex with strong correlation between $\theta$ and $\varphi$ [see Fig. 1(b) and (e)]. In ferromagnetic metals, $\beta(\sim 0.001-0.01)$ is small ${ }^{10,30}$ and $A_{s s}^{0}$ is large due to the strong dipole-dipole interaction, hence the vertical spin stiffness is unimportant. However, in ferromagnetic semiconductors, e.g., GaMnAs, $A_{\mathrm{ss}}^{0}$ would be small ${ }^{31,32}$ and $\beta$ can be large, $\beta^{\prime} \approx \beta \sim 1, \stackrel{20,23}{2}$ therefore, the domain wall structure can behave like a vortex.

When $K_{\perp} \gtrsim 1$, the behavior of $\varphi$ in a steady solution is determined by the competition between the hard-axis anisotropy, $K_{\perp}$, and the transverse stiffness is proportional to $\beta^{\prime}$. Then $\varphi$ tends to be locked to $\varphi=n \pi(n$ is integer) and deviates from being a linear function of position, forming soliton-like structure or a staircase behavior [Fig.11(c) and (f)]. In a domain wall, modulation of $\theta$ occurs when $\phi$ is close to $n \pi$, but $\theta$ tends to be close to $n^{\prime} \pi$ ( $n^{\prime}$ is integer) when $\varphi$ changes in order to lower the energy cost due to the hard axis anisotropy. Thus a domain wall has an oscillating structure as seen in Fig.1(c) and (f). The number of oscillation increases as $\beta^{\prime}$ is enhanced. As far as we find numerically, the number of oscillation also depends on the initial condition of $\partial_{x} \theta$ and $\partial_{x} \varphi$ at the boundary of the wall. Finally, we should point out that the domain-wall solution for $K_{\perp} \neq 0$ is the same as that for $K_{\perp}=0$ in the absence of the vertical spin stiffness [see Fig. 1(a) and (d)] $\underline{29}$

As is well-known,,$\frac{4}{\underline{-}}$ the dynamics is strongly affected by the structure. For instance, vortex walls are easier to move than planar domain walls in the current-driven case, because of the perpendicular component near the vortex core. We may therefore expect that the wall for finite $\beta^{\prime} \gtrsim 1$, e.g., in GaMnAs, $\stackrel{20}{,}$ would have even lower threshold current due to the structure change arising from the transverse exchange torque. Inclusion of the transverse stiffness in the micromagnetic simulations is thus crucially important in systems with strong spinorbit interaction.

\section{SUMMARY}

In summary, we have derived the KSBEs in ferromagnetic systems based on the $s$ - $d$ model in the presence of the inhomogeneity of the magnetization. We analytically solved the KSBEs and derived the spin torque due to the spin polarization of the itinerant electrons. The current-induced spin torque from the first-order magnetization gradient is consistent with the previous works. We found that the second-order gradient of the magnetization inhomogeneity gives rise to an effective magnetic field that is perpendicular to the spin stiffness field. This vertical spin stiffness is proportional to the nonadiabatic parameter $\beta$. We showed that the new term modifies the domain wall structure and causes magnetization rotation along the easy axis. The vertical spin stiffness is expected to be crucially important in ferromagnetic semiconductors, and needs to be included in the LLG equation in numerical simulations on the magnetization dynamics.

\section{Acknowledgments}

This work was supported by the Natural Science Foundation of China under Grant No. 10725417. One of the authors (G.T.) thanks support by a Grant-in-Aid for Scientific Research in Priority Areas, "Creation and control of spin current" (Grant No. 1948027), the Kurata Memorial Hitachi Science and Technology Foundation, and the Sumitomo Foundation.

\section{Appendix A: Fourier transformation of Eq. (7)}

The Fourier transformations of the functions in Eq. (7) respect to the relative coordinate are calculated as follows. For simplification, we omit all the temporal coordinates without leading to any ambiguity.

The Green function at momentum $\mathbf{k}$ is defined as

$$
G(\mathbf{R}, \mathbf{k})=\int d \mathbf{r} e^{-i \mathbf{k} \cdot \mathbf{r}} G(\mathbf{R}, \mathbf{r}) .
$$

Then, the electric potential energy term can be written as

$$
\begin{aligned}
& \int d \mathbf{r} e^{-i \mathbf{k} \cdot \mathbf{r}} U_{e}(1) G(\mathbf{R}, \mathbf{r}) \\
& =\int d \mathbf{r} e^{-i \mathbf{k} \cdot \mathbf{r}} e^{\frac{\mathbf{r}}{2} \partial_{\mathbf{R}}^{U}} U_{e}(\mathbf{R}) G(\mathbf{R}, \mathbf{r}) \\
& =e^{i \frac{1}{2} \partial_{\mathbf{k}} \partial_{\mathbf{R}}^{U e}} \int d \mathbf{k} e^{-i \mathbf{k} \cdot \mathbf{r}} U_{e}(\mathbf{R}) G(\mathbf{R}, \mathbf{r}) \\
& =e^{i \frac{1}{2} \partial_{\mathbf{k}}^{G} \partial_{\mathbf{R}}^{U e}} U_{e}(\mathbf{R}) G(\mathbf{R}, \mathbf{k}) .
\end{aligned}
$$

Terms with Hamiltonian read

$$
\begin{aligned}
& \int d \mathbf{r} e^{-i \mathbf{k} \cdot \mathbf{r}} H_{0}\left(\mathbf{p}_{1}, \mathbf{r}_{1}\right) G(\mathbf{R}, \mathbf{r}) \\
& \quad=\int d \mathbf{r} e^{-i \mathbf{k} \cdot \mathbf{r}} e^{\frac{\mathbf{r}}{2} \partial_{\mathbf{R}}^{H_{0}}} H_{0}\left(\frac{1}{2} \mathbf{P}_{\mathbf{R}}+\mathbf{p}, \mathbf{R}\right) G(\mathbf{R}, \mathbf{r}) \\
& =e^{i \frac{1}{2} \partial_{\mathbf{k}} \partial_{\mathbf{R}}^{H_{0}}} \int d \mathbf{r} e^{-i \mathbf{k} \cdot \mathbf{r}} H_{0}\left(\frac{1}{2} \mathbf{P}_{\mathbf{R}}+\mathbf{p}, \mathbf{R}\right) G(\mathbf{R}, \mathbf{r}) \\
& =e^{i \frac{1}{2} \partial_{\mathbf{k}} \partial_{\mathbf{R}}^{H_{0}}} H_{0}\left(\frac{1}{2} \mathbf{P}_{\mathbf{R}}+\mathbf{k}, \mathbf{R}\right) G(\mathbf{R}, \mathbf{k}) \\
& =e^{i \frac{1}{2}\left(\partial_{\mathbf{k}} \partial_{\mathbf{R}}^{H_{0}}-\partial_{\mathbf{R}}^{G} \partial_{\mathbf{k}}^{H_{0}}\right)} H_{0}(\mathbf{k}, \mathbf{R}) G(\mathbf{R}, \mathbf{k}),
\end{aligned}
$$

and

$$
\begin{aligned}
\int & d \mathbf{r} e^{-i \mathbf{k} \cdot \mathbf{r}}\left[G(\mathbf{R}, \mathbf{r}) H_{0}\left(-\overleftarrow{\mathbf{p}}_{2}, \mathbf{r}_{2}\right)\right] \\
& =\int d \mathbf{r} e^{-i \mathbf{k} \cdot \mathbf{r}} e^{-\frac{\mathbf{r}}{2} \partial_{\mathbf{R}}^{H_{0}}}\left[G(\mathbf{R}, \mathbf{r}) H_{0}\left(-\frac{1}{2} \overleftarrow{\mathbf{P}}_{\mathbf{R}}+\overleftarrow{\mathbf{p}}, \mathbf{R}\right)\right] \\
& =e^{-i \frac{1}{2} \partial_{\mathbf{k}} \partial_{\mathbf{R}}^{H_{0}}} \int d \mathbf{r} e^{-i \mathbf{k} \cdot \mathbf{r}}\left[G(\mathbf{R}, \mathbf{r}) H_{0}\left(-\frac{1}{2} \overleftarrow{\mathbf{P}_{\mathbf{R}}}+\overleftarrow{\mathbf{p}}, \mathbf{R}\right)\right] \\
& =e^{-i \frac{1}{2} \partial_{\mathbf{k}} \partial_{\mathbf{R}}^{H_{0}}} G(\mathbf{R}, \mathbf{k}) H_{0}\left(-\frac{1}{2} \overleftarrow{\mathbf{P}_{\mathbf{R}}}+\mathbf{k}, \mathbf{R}\right) \\
& =e^{-i \frac{1}{2}\left(\partial_{\mathbf{k}} \partial_{\mathbf{R}}^{H_{0}}-\partial_{\mathbf{R}}^{G} \partial_{\mathbf{k}}^{H_{0}}\right)} G(\mathbf{R}, \mathbf{k}) H_{0}(\mathbf{k}, \mathbf{R})
\end{aligned}
$$


For the integral terms, one has

$$
\begin{aligned}
\int d \mathbf{r} & e^{-i \mathbf{k} \cdot \mathbf{r}} \int d \mathbf{r}_{3} \Sigma(1,3) G(3,2) \\
= & \int d \mathbf{r} e^{-i \mathbf{k} \cdot \mathbf{r}} \int d \mathbf{r}_{3} e^{\frac{\mathbf{r}_{3}-\mathbf{r}_{2}}{2} \partial_{\mathbf{R}}^{\Sigma}} \Sigma\left(\mathbf{R}, \mathbf{r}_{1}-\mathbf{r}_{3}\right) \\
& \times e^{\frac{\mathbf{r}_{3}-\mathbf{r}_{1}}{2} \partial_{\mathbf{R}}^{G}} G\left(\mathbf{R}, \mathbf{r}_{3}-\mathbf{r}_{2}\right) \\
= & \int d \mathbf{r} e^{-i \mathbf{k} \cdot \mathbf{r}} \int d \mathbf{r}_{3} e^{\frac{\mathbf{r}_{3}-\mathbf{r}_{2}}{2} \partial_{\mathbf{R}}^{\Sigma}} \int \frac{d \mathbf{k}}{(2 \pi)^{3}} e^{i \mathbf{k}^{\prime} \cdot\left(\mathbf{r}_{1}-\mathbf{r}_{3}\right)} \Sigma\left(\mathbf{R}, \mathbf{k}^{\prime}\right) \\
= & \int d e^{\frac{\mathbf{r}_{3}-\mathbf{r}_{1}}{2} \partial_{\mathbf{R}}^{G}} \int \frac{d \mathbf{k}_{3}^{\prime \prime}}{(2 \pi)^{3}} e^{i \mathbf{k}^{\prime \prime} \cdot\left(\mathbf{r}_{3}-\mathbf{r}_{2}\right)} G\left(\mathbf{R}, \mathbf{k}^{\prime \prime}\right) \\
& \quad \frac{d \mathbf{k}^{\prime \prime}}{(2 \pi)^{3}} e^{-i \mathbf{k} \cdot \mathbf{r}}\left[e^{-\frac{i}{2} \partial_{\mathbf{k}^{\prime \prime}} \partial_{\mathbf{R}}^{\Sigma}} e^{\frac{i}{2} \partial_{\mathbf{k}^{\prime}} \partial_{\mathbf{R}}^{G}} e^{i \mathbf{k}^{\prime} \cdot\left(\mathbf{r}_{1}-\mathbf{r}_{3}\right)}\right. \\
& \left.\times e^{i \mathbf{k}^{\prime \prime} \cdot\left(\mathbf{r}_{3}-\mathbf{r}_{2}\right)}\right] G\left(\mathbf{R}, \mathbf{k}^{\prime}\right) \Sigma\left(\mathbf{R}, \mathbf{k}^{\prime \prime}\right) \\
= & e^{\frac{i}{2}\left(\partial_{\mathbf{k}}^{G} \partial_{\mathbf{R}}^{\Sigma}-\partial_{\mathbf{k}}^{\Sigma} \partial_{\mathbf{R}}^{G}\right)} \Sigma(\mathbf{R}, \mathbf{k}) G(\mathbf{R}, \mathbf{k}) .
\end{aligned}
$$

Here, the time integral $\int d t_{3}$ is omitted for simplification. Similarly, one can show

$$
\begin{aligned}
& \int d \mathbf{r} e^{-i \mathbf{k} \cdot \mathbf{r}} \int d \mathbf{r}_{3} \Sigma(1,3) G(3,2) \\
& =e^{\frac{i}{2}\left(\partial_{\mathbf{k}}^{\Sigma} \partial_{\mathbf{R}}^{G}-\partial_{\mathbf{k}}^{G} \partial_{\mathbf{R}}^{\Sigma}\right)} G(\mathbf{R}, \mathbf{k}) \Sigma(\mathbf{R}, \mathbf{k}) .
\end{aligned}
$$

In these equations, we use the notation $\partial_{\mathbf{R}} \partial_{\mathbf{k}}=\nabla_{\mathbf{R}} \cdot \nabla_{\mathbf{k}}$.
* Author to whom correspondence should be addressed; Electronic address: mwwu@ustc.edu.cn.

1 C. Kittel, Rev. Mod. Phys. 21, 541 (1949).

2 A. Hubert and R. Schäfer, Magnetic Domains (SpringerVerlag, Berlin, 1998)

3 Y. Tserkovnyak, A. Brataas, G. E. W. Bauer, and B. I. Halperin, Rev. Mod. Phys. 77, 1375 (2005).

${ }^{4}$ G. Tatara, H. Kohno, and J. Shibata, Phys. Rep. 468, 213 (2008).

5 D. A. Allwood, G. Xiong, C. C. Faulkner, D. Atkinson, D. Petit, and R. P. Cowburn, Science 309, 1688 (2005).

${ }^{6}$ L. Thomas, M. Hayashi, X. Jiang, R. Moriya, C. Rettner, and S. S. P. Parkin, Nature (London) 443, 197 (2006).

7 S. S. P. Parkin, M. Hayashi, and L. Thomas, Science 320, 190 (2008).

8 T. L. Gilbert, Phys. Rev. 100, 1243 (1955).

${ }^{9}$ L. D. Landau, E. M. Lifshitz, and L. P. Pitaevski, Statistical Physics, Part 2, 3rd ed. (Pergamon, Oxford, 1980).

10 Y. Tserkovnyak, A. Brataas, and G. E. W. Bauer, J. Magn. Magn. Mater. 320, 1282 (2008).

11 L. Berger, Phys. Rev. B 33, 1572 (1986).

12 S. Zhang and Z. Li, Phys. Rev. Lett. 93, 127204 (2004).

13 A. Thiaville, Y. Nakatani, J. Miltat, and Y. Suzuki, Europhys. Lett. 69, 990 (2005).

14 H. Kohno, G. Tatara, and J. Shibata, J. Phys. Soc. Jpn. 75, 113706 (2006).

15 H. Kohno and J. Shibata, J. Phys. Soc. Jpn. 76, 063710 (2007).

16 Y. Tserkovnyak, H. J. Skadsem, A. Brataas, and G. E. W. Bauer, Phys. Rev. B 74, 144405 (2006).

17 Z. Li and S. Zhang, Phys. Rev. B 70, 024417 (2004).

18 G. Tatara and H. Kohno, Phys. Rev. Lett. 92, 086601
(2004).

19 G. Tatara, T. Takayama, H. Kohno, J. Shibata, Y. Nakatani, and H. Fukuyama, J. Phys. Soc. Jpn. 75, 064708 (2006).

${ }^{20}$ K. M. D. Hals, A. K. Nguyen, and A. Brataas, Phys. Rev. Lett. 102, 256601 (2009).

${ }^{21}$ K. Shen, G. Tatara, and M. W. Wu, Phys. Rev. B 81, 193201 (2010).

22 M. W. Wu, J. H. Jiang, and M. Q. Weng, Phys. Rep. 493, 61 (2010), and references therein.

23 C. M. Jaworshi, J. Yang, S. Mack, D. D. Awschalom, J. P. Heremans, and R. C. Myers, Nature Mater. 9, 898 (2010).

24 E. L. Nagaev, Physics of Magnetic Semiconductors (Mir, Moscow, 1983).

25 J. König, H. H. Lin, and A. H. MacDonald, Phys. Rev. Lett. 84, 5628 (2000).

26 J. König, T. Jungwirth, and A. H. MacDonald, Phys. Rev. B 64, 184423 (2001).

27 H. Haug and A.-P. Jauho, Quantum Kinetics in Transport and Optics of Semiconductors (Springer-Verlag, Berlin, 1996).

28 M. W. Wu and H. Metiu, Phys. Rev. B 61, 2945 (2000).

29 N. L. Schryer and L. R. Walker, J. Appl. Phys. 45, 5406 (1974).

30 D. C. Ralph and M. D. Stiles, J. Magn. Magn. Mater. 320, 1190 (2008).

31 T. Dietl, H. Ohno, and F. Matsukura, Phys. Rev. B 63, 195205 (2001).

32 L. Cywiński and L. J. Sham, Phys. Rev. B 76, 045205 (2007). 